though the prevalence of brain tumor was $0.5 \%$ (range 0-2\%). Except for a missed diagnosis of volvulus resulting in extensive small-bowel resection, the cost of delay in diagnosis of other causes of cyclic vomiting (eg metabolic) were less than the diagnostic tests. (Olson AD, Li BUK. The diagnostic evaluation of children with cyclic vomiting: a cost-effectiveness assessment. I Pediatr November 2002;141:724728). (Reprints: Allan D Olson MD, MBA, Centocor Inc, 200 Great Valley Parkway, Malvern, PA 19355).

COMMENT. According to these authors and based on cost-effectiveness, the most practical approach to the diagnosis of cyclic vomiting $(\mathrm{CV})$ is an initial smallbowel radiograph to rule out malrotation followed by a 2 -month empiric trial of antimigraine medication. A positive family history of migraine is reported in $82 \%$ of patients with idiopathic $\mathrm{CV}$, and their response to migraine prophylaxis is better than those without a family history of migraine (79\% vs 36\%). (Li B. Pediatrics 1998;102:583-587). Only 12\% have serious underlying disorders such as intestinal malrotation with volvulus, brain tumors, or metabolic disorders.

Cyclic vomiting is reported as a form of epilepsy in children (Millichap JG et al. Pediatrics 1955;15:705-712), and ictus emeticus with nondominant temporal lobe involvement is a well documented form of autonomic epilepsy, sometimes induced by photic stimulation (Guerrini R et al. Neurology 1994;44:253-259). See Progress in Pediatric Neurology I \& III, 1991 \& 1997, for further review of ictus emeticus.

Vomiting as an ictal phenomenon is controversial and difficult to distinguish from migraine. Symptoms should be paroxysmal and associated with ictal epileptiform discharges on the EEG. In our own retrospective study of 33 children with cyclic vomiting reported in 1955, $25(76 \%)$ had interictal seizure discharges in the EEG, some focal with temporal localization, and 7 (21\%) had a history of complex partial or generalized seizures. Epilepsy should certainly be considered in the differential diagnosis of cyclic vomiting, an omission in the above report, and an EEG obtained, if possible during a vomiting episode. A history of previous seizures and family history of epilepsy, brain pathology, and a beneficial response to antiepileptic drugs will help to corroborate the diagnosis. An abnormal EEG is common in children with migraine, and AEDs are an effective migraine prophylaxis (Millichap JG. Child's Brain 1978;4:95-105), a further confounding factor in the diagnosis of cyclic vomiting and differentiation of an epilepsy or migraine.

\title{
KLEINE-LEVIN SYNDROME: AN AUTOIMMUNE HYPOTHESIS
}

Clinical, polysomnographic, CSF, CT, and MRI records and genotype data of 30 unrelated patients with Kleine-Levin syndrome (KLS) and their families were studied at Hopital Gui-de-Chauliac, Montpellier, France, and other sleep centers. The sex ratio showed a predominance of males $(25 / 5)$. The mean age at onset was $15+/-3$ years. The mean number of episodes per year was $4+/-3$ and the mean duration of episodes was $12+/-8$ days. Viral upper respiratory tract infection was a precipitating factor in 16/23 (70\%). The two essential diagnostic criteria, recurrent hypersomnia with asssociated cognitive and mood disturbances, were present in all patients. Hyperphagia occurred in 17 (57\%), and hypersexuality in 14 (47\%) patients. Brain imaging and CSF were normal. EEGs were not recorded. Treatment for hypersomnia that was partially effective included the stimulant, modafinil, carbamazepine, and lithium. Neuroleptics were ineffective. The genotype data analysis of KLS patients, contrasted with normal controls, found a significant increase in the frequency of human leukocyte antigen (HLA)$\mathrm{DQB1}{ }^{\star} 0201$ allele, and 3 patients with KLS were homozygous for DQB1*0201, 2 from 
the same family. An autoimmune etiology is suggested. (Dauvilliers Y, Mayer G, Lecendreux $\mathrm{M}$ et al. Kleine-Levin syndrome: an autoimmune hypothesis based on clinical and genetic analyses. Neurology December (1 of 2) 2002;59:1739-1745). (Reprints: Dr Mehdi Tafti, HUG, Belle-Idee, Biochemistry and Genetics, Chemin du Petit-BelAir, 2, CH-1225 Chene-Bourg, Switzerland).

COMMENT. The etiology of Kleine-Levin syndrome is unknown. Periodic hypothalamic dysfunction, focal encephalitis, and an autoimmune process are possibilities. The disorder is rarely familial, only in 2 of 30 patients in the above study, and in 2 siblings in the following report. Levels of CSF orexin (hypocretin), decreased in some narcoleptic patients, were normal in the following familial cases.

Familial Kleine-Levin syndrome is reported in 2 siblings with unusually long hypersomnic spells, from Tufts University, Boston, MA (Katz JD, Ropper AH. Arch Neurol Dec 2002;59:1959-1961). Both patients shared the HLA-DR2, DQ1, and DR5 haplotypes. Treatment with lithium, methylphenidate, modafinil, clonazepam, and other drugs was ineffective in preventing or reducing the duration of hypersomnic spells. Patient 1, a 15-year-old boy, developed flu-like symptoms without fever, he slept excessively for 9 days, and was irritable when awake. Eight similar attacks occurred during the following 10 months, and 16 hypersomnic spells in 5 years. Compulsive symptoms followed the episodes. Patient 2 , the sister of patient 1 , began having hypersomnic spells at age 13 years, the first preceded by a flu-like illness. Between attacks, MRI, EEG, Ebstein-Barr virus titer, ESR, and CSF were normal.

\section{VASCULAR DISORDERS}

\section{TIME LAG TO DIAGNOSIS OF STROKE}

Time to diagnosis was determined in 41 children, 0 to 18 years, with ischemic or hemorrhagic stroke documented prospectively or by retrospective chart review of the last 2 years database, at State University of New York, Stony Brook, NY. Twelve neonates diagnosed in the neonatal period were excluded from analysis. Of the remaining 29 (mean age at presentation 8.67 years), 24 had accurate time records, and 28 events were recorded. Ischemic stroke occurred in 21 events and hemorrhagic stroke in 7. The cause was idiopathic in 18 (46\%) and embolic in 4 (surgery and atrial myxoma); vascular malformation was present in 3 , arterial dissection in 2 , inherited coagulopathy in 3 , and moyamoya or other syndrome in 4. An underlying known disorder was found in $38 \%$, and a predisposing disorder in an additional 15\%. Initial symptoms were headache in $32 \%$, motor in $60 \%$, sensory in $7 \%$, aphasia in $14 \%$, seizures in $10 \%$, and mental status change in $21 \%$. Time from clinical onset to first medical exam was an average of 28.5 hours, and time to diagnosis of stroke averaged 35.7 hours. Unless hemorrhagic, stroke in children is rarely diagnosed in $<3$ to 6 hours from onset, a major criterion for inclusion in therapeutic trials of thrombolytic or neuroprotective agents. (Gabis LV, Yangala R, Lenn NJ. Time lag to diagnosis of stroke in children. Pediatrics November 2002;110:924-928). (Reprints: Lidia V Gabis MD, Division of Developmental Disabilities, South Campus, Putnam Hall, State University of New York, Stony Brook, NY 11794).

COMMENT. Stroke with massive hemorrhage demands immediate medical attention. Ischemic stroke is less dramatic and medical attention is delayed. Compared to the 35 hour delay in children, stroke in adults is treated as an 